\title{
Inversion of distance and magnetic permeability based on material-independent and lift-off insensitive algorithms using eddy current sensor
}

\author{
Mingyang Lu*, Xiaobai Meng, Ruochen Huang*, Liming Chen, Anthony Peyton, Wuliang Yin*
}

\begin{abstract}
Eddy current sensors can be used to test the characteristics and measure the parameters of the conductive samples. As the main obstacle of the multi-frequency eddy current sensor, the lift-off distance affects the effectiveness and accuracy of the measurement. In this paper, a material-independent algorithm has been proposed for the restoration of the lift-off distance when using the multi-frequency eddy current sensor, which is based on the approximation under the thin-skin effect. Experiment testing on the performance of the proposed method is presented. Results show that from the dual-frequency inductance, the lift-off distance could be restored with a maximum error of $0.24 \mathrm{~mm}$ for the distance up to $12 \mathrm{~mm}$. Besides, the derived lift-off distance is used for the inversion of the magnetic permeability. Based on a lift-off insensitive inductance (LII) feature, the magnetic permeability of steels can be inversed in an iterative manner, with an error of less than $0.6 \%$ for the lift-off distance up to $12 \mathrm{~mm}$.
\end{abstract}

Index Terms - Eddy current; lift-off; material-independent; permeability measurement; non-destructive testing.

\section{INTRODUCTION}

E DDY current techniques are widely used in interrogating the conductive materials in diverse industrial nondestructive testing (NDT) [1-9]. Owing to its merits (including high adaptability and sensitivity), the eddy current sensor has been used for the testing of the material characteristics, detection of structural integrity, the inspection of surface crack fatigue, and measurement of material properties including thickness and electromagnetic (EM) properties (electrical conductivity and magnetic permeability). The EM properties of materials are directly linked to the phase fractions of alloys [4]. To increase material homogeneity, and thus improve consistency in the mechanical properties, significant advances in materials characterisation would be obtained if the EM property information could be determined online during steel production in a non-destructive and remote manner [4]. However, like other eddy current techniques (including the single-frequency eddy current testing and pulsed eddy current testing), the multi-frequency eddy current (MEC) testing is sensitive to the lift-off distance between the sensor and test piece (particularly for the surface-defected sample), which

This work was supported by [UK Engineering and Physical Sciences Research Council (EPSRC)] [grant number: EP/P027237/1] [title: Real-time Inline Microstructural Engineering (RIME)].

M. Lu (Member, IEEE), R. Huang, L. Chen, A. Peyton and W. Yin (Senior Member, IEEE) are with the School of Electrical and Electronic Engineering, University of Manchester, Sackville Street Building, Manchester, M13 9PL, could influence the accuracy of the measurement [10-15].

To address the lift-off issue, strategies including optimization of the coil structure, signal processing techniques, and novel measurement methods have been proposed. By analysing the signature of receiving coils, Giguere et al. have found a lift-off point of intersection (LOI) feature using the PEC method [16]. The exploited LOI feature does not vary significantly with variation in coupling or increase in probe liftoff. Researchers have further optimized, polished, and implemented the LOI feature for the measurement of materials using PEC techniques [17]. Abu-Nabah has proposed a semiquadratic system to reduce the lift-off effect in high-frequency apparent eddy current conductivity spectroscopy [18]. Moreover, a phase signature has been used by Yin et al and Pinotti et al from the multi-frequency inductance [19-21]. With the proposed phase signature, the inductance change caused by the test sample is less affected by the lift-off distance. However, it has been found the inductance phase is still sensitive to the lift-off around the inflection point (near to the peak frequency feature) [22]. Therefore, an algorithm has been proposed for compensating the lift-off noise of the impedance/inductance phase [22]. However, compared to the impedance or inductance, its phase is less sensitive to the test piece. Therefore, it is necessary to explore an alternative feature from the swept inductance instead of its phase.

Previously, to reduce the lift-off effect, approaches involve planar sensor designs, multi-frequency features (including zerocrossing feature, and peak frequency features), the lift-off invariant phenomenon (conductivity and permeability invariant phenomena), and the phase signature [22-30]. In this paper, an alternative method based on the material-independent phase term under the eddy-current thin-skin effect has been proposed, which has improved and extended the previous measurement range of the lift-off distance (from $6 \mathrm{~mm}$ to $12 \mathrm{~mm}$ ) without sacrificing the accuracy. Experiment testing on different magnetic steels has been carried out. The lift-off distance can be restored from the inductance of dual high frequencies, which is shown independent of different materials. Moreover, an identical lift-off insensitive inductance (LII) feature has been found on the swept frequency inductance of different magnetic

UK (mingyang.lu@manchester.ac.uk; ruochen.huang@manchester.ac.uk; wuliang.yin@manchester.ac.uk)

*Corresponding author: M. Lu, R. Huang, W. Yin.

X. Meng is with the Faculty of Art, Science and Technology, University of Northampton, Northampton, NN1 5PH, UK 
steels. That is, multi-frequency inductance curves of different lift-offs nearly intersect on one point. By referring to the restored lift-off and the corresponding frequency of LII, the relative magnetic permeability of ferromagnetic steels has been retrieved.

\section{MATERIAL-INDEPENDENT ALGORITHM AND LIFT-OFF INSENSITIVE INDUCTANCE FEATURE}

\section{A. Original analytical formulation}

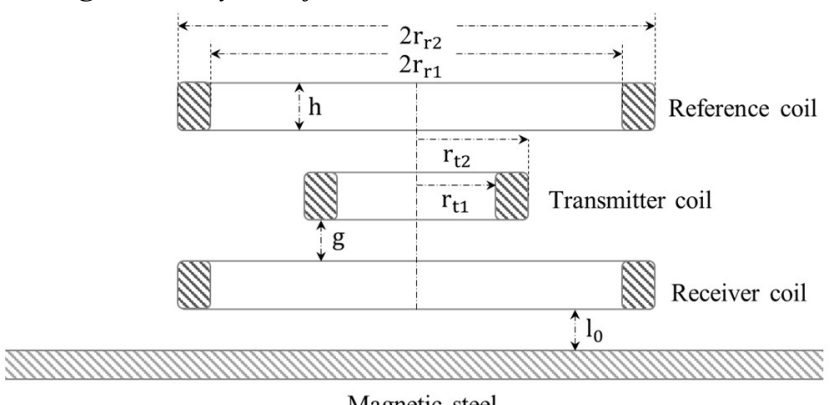

Magnetic steel

Fig. 1 Eddy current sensor consist of three co-axial coil windings

As shown in Fig. 1, the eddy current sensor is composed of three co-axial coil windings. Since the magnetic flux generated from the transmitter diverges for a considerable distance (the gap, $g$ between the transmitter and receiver, as shown in Fig. 1 and Table 1), particularly for a relatively large lift-off distance between the sensor and sample, the radius of the receiver and reference coil is designed larger than that of the transmitter to fully catch the reflected magnetic flux.

In recent years, the Dodd-Deeds formula [31] has been widely used for the inductance analysis of the air-core coil above the conductive plates [32-37]. For the designed eddy current sensor shown in Fig. 1, the inductance change caused by the test magnetic steel is given by the expression.

$$
\begin{aligned}
& \mathrm{L}_{1}=\mathrm{K} \int_{0}^{\infty} \mathrm{M}_{1} \mathrm{e}^{-2 \alpha \mathrm{l}_{0}} \Phi \mathrm{d} \alpha \\
& \mathrm{L}_{2}=\mathrm{K} \int_{0}^{\infty} \mathrm{M}_{2} \mathrm{e}^{-2 \alpha \mathrm{l}_{0}} \Phi \mathrm{d} \alpha
\end{aligned}
$$

$\mathrm{L}_{1}$ and $\mathrm{L}_{2}$ are the inductance change from the transmitterreceiver and transmitter-reference sensing pairs. $\mathrm{K}$ is a constant related to the cross-section of the coil.

$$
K=\frac{\pi \mu_{0} N^{2}\left(r_{r 2}+r_{r 1}\right)}{2 h^{2}\left(r_{r 2}-r_{r 1}\right)\left(r_{t 2}-r_{t 1}\right)}
$$

In (3), $\mu_{0}$ is the magnetic permeability of the free space (or vacuum magnetic permeability). Three coils have identical coil heights $\mathrm{h}$, winding turns $\mathrm{N}$, and separation distance $\mathrm{g}$. The inner and outer radius of the transmitter coil is $r_{t 1}$ and $r_{t 2}$. The receiver and reference coil have the same inner and outer radius $r_{r 1}$ and $r_{r 2}$. The integrand consists of the coil-dependent magnitude part $\left(\mathrm{M}_{1}\right.$ and $\left.\mathrm{M}_{2}\right)$ with the lift-off decay term $\left(\mathrm{e}^{-2 \alpha \mathrm{l}_{0}}\right)$, and the material-dependent phase term $(\Phi)$.

$$
\begin{aligned}
& \mathrm{M}_{1}=\frac{\mathrm{P}_{\mathrm{t}} \mathrm{P}_{\mathrm{r}}}{\alpha^{6}} \mathrm{e}^{-\alpha(\mathrm{h}+\mathrm{g})}\left(\mathrm{e}^{-\alpha \mathrm{h}}-1\right)^{2} \\
& \mathrm{M}_{2}=\frac{\mathrm{P}_{\mathrm{t}} \mathrm{P}_{\mathrm{r}}}{\alpha^{6}} \mathrm{e}^{-3 \alpha(\mathrm{h}+\mathrm{g})}\left(\mathrm{e}^{-\alpha \mathrm{h}}-1\right)^{2}
\end{aligned}
$$

where $P_{t}$ and $P_{r}$ are the integral of the Bessel series; $\alpha$ is related to the wavenumber of the incident transverse electric
(TE) plane EM wave (in the free space) [31,37].

$$
\begin{aligned}
& P_{t}=\int_{\alpha r_{t 1}}^{\alpha r_{t 2}} \tau J_{1}(\tau) d \tau \\
& P_{r}=\int_{\alpha r_{r 1}}^{\alpha r_{r 2}} \tau J_{1}(\tau) d \tau
\end{aligned}
$$

$\mathrm{J}_{1}$ denotes the first-order Bessel function of the first kind.

The material-dependent phase term $(\Phi)$ is defined as the real part of a complex fractional function.

$$
\Phi=\operatorname{Re}\left(\frac{\left(\alpha_{1}+\mu_{1} \alpha\right)\left(\alpha_{1}-\mu_{1} \alpha\right)-\left(\alpha_{1}+\mu_{1} \alpha\right)\left(\alpha_{1}-\mu_{1} \alpha\right) \mathrm{e}^{2 \alpha_{1} c}}{-\left(\alpha_{1}-\mu_{1} \alpha\right)\left(\alpha_{1}-\mu_{1} \alpha\right)+\left(\alpha_{1}+\mu_{1} \alpha\right)\left(\alpha_{1}+\mu_{1} \alpha\right) \mathrm{e}^{2 \alpha_{1} c}}\right)
$$

$\mu_{1}$ and $c$ are the relative magnetic permeability and thickness of the magnetic plate. $\alpha_{1}$ is the square root of a complex term, which is related to the wavenumber of the transverse electric (TE) plane EM wave (in the steel) $[31,38]$.

$$
\alpha_{1}=\sqrt{\alpha^{2}+j 2 \pi \sigma \mu_{1} \mu_{0} \mathrm{f}}
$$

$\mathrm{f}$ is the working frequency of the exciting current.

\section{B. Integration version of material-independent algorithm- inversion of lift-off distance}

Generally, for a magnetic steel slab (unlike the non-magnetic materials), the eddy current is restrained around the surface of the sample even under the working frequency of $100 \mathrm{~Hz}$ (referring to the skin depth formula $\left(\pi \sigma \mu_{0} f\right)^{-1 / 2}$ ). Owing to the eddy current skin effect, the magnetic slab can be treated as a conductive half-space. Therefore, $\operatorname{Re}\left(\mathrm{e}^{2 \alpha_{1} c}\right) \gg 1$ satisfies. Besides, $\Phi$ in (8) can be expressed as

$$
\Phi=\operatorname{Re}\left(\frac{\mu_{1} \alpha-\alpha_{1}}{\mu_{1} \alpha+\alpha_{1}}\right)
$$

For an eddy current sensor with moderate size (e.g. Table 1) the effective range of $\alpha$ is limited (according to Fig. 3, from 0 to 180 for the sensor in Table 1). That is, $2 \pi \sigma \mu_{1} \mu_{0} f \gg \alpha^{2}$ for the whole frequency range. Thus, (10) can be simplified as

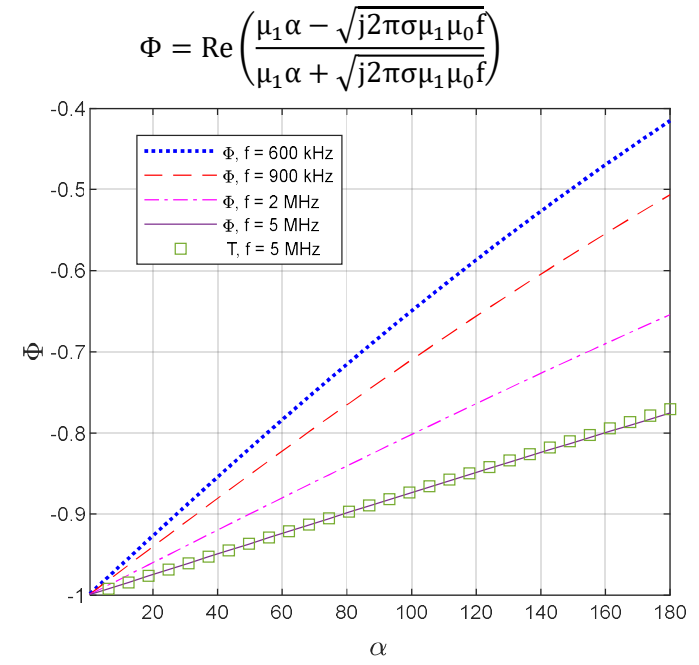

Fig. $2 \Phi$ and simplified linear function $\mathrm{T}$ under high working frequencies

As shown in Fig. 2, for the relatively high working frequencies (particularly frequencies exceed $400 \mathrm{kHz}$ ), it has been found $\Phi$ can be approximated with a linear function $T$.

$$
\mathrm{T}=\sqrt{\frac{\mu_{1}}{\pi \sigma \mu_{0} \mathrm{f}}} \alpha-1
$$

In (12), the slope is related to material-dependent parameters $\mu_{1}, \sigma$, and the working frequency $\mathrm{f}$. Thus, the inductance 
formula in (1) and (2) can be expressed as two terms, with one dependent on the material and working frequency.

$$
\begin{aligned}
& \mathrm{L}_{1}=\mathrm{K} \sqrt{\frac{\mu_{1}}{\pi \sigma \mu_{0} \mathrm{f}}} \int_{0}^{\infty} \alpha \mathrm{M}_{1} \mathrm{e}^{-2 \alpha \mathrm{l}_{0}} \mathrm{~d} \alpha-\mathrm{K} \int_{0}^{\infty} \mathrm{M}_{1} \mathrm{e}^{-2 \alpha \mathrm{l}_{0}} \mathrm{~d} \alpha \\
& \mathrm{L}_{2}=\mathrm{K} \sqrt{\frac{\mu_{1}}{\pi \sigma \mu_{0} \mathrm{f}}} \int_{0}^{\infty} \alpha \mathrm{M}_{2} \mathrm{e}^{-2 \alpha \mathrm{l}_{0}} \mathrm{~d} \alpha-\mathrm{K} \int_{0}^{\infty} \mathrm{M}_{2} \mathrm{e}^{-2 \alpha \mathrm{l}_{0}} \mathrm{~d} \alpha
\end{aligned}
$$

From (13) and (14), the material-dependent term (first term of right sides) can be eliminated as,

$$
\mathrm{Y}_{2}\left(\mathrm{~L}_{1}+\mathrm{KF}_{1}\right)-\mathrm{Y}_{1}\left(\mathrm{~L}_{2}+\mathrm{KF}_{2}\right)=0
$$

In (15), $F_{1}, F_{2}, Y_{1}$, and $Y_{2}$ are only dependent on the parameter of the sensor and lift-off variation $\mathrm{l}_{0}$.

$$
\begin{aligned}
& F_{1}=\int_{0}^{\infty} \mathrm{M}_{1} \mathrm{e}^{-2 \alpha \mathrm{l}_{0}} \mathrm{~d} \alpha \\
& \mathrm{F}_{2}=\int_{0}^{\infty} \mathrm{M}_{2} \mathrm{e}^{-2 \alpha \mathrm{l}_{0}} \mathrm{~d} \alpha \\
& \mathrm{Y}_{1}=\int_{0}^{\infty} \alpha \mathrm{M}_{1} \mathrm{e}^{-2 \alpha \mathrm{l}_{0}} \mathrm{~d} \alpha \\
& \mathrm{Y}_{2}=\int_{0}^{\infty} \alpha \mathrm{M}_{2} \mathrm{e}^{-2 \alpha \mathrm{l}_{0}} \mathrm{~d} \alpha
\end{aligned}
$$

Theoretically, with the measured inductance from transmitter-receiver $\mathrm{L}_{1}$ and transmitter-reference $\mathrm{L}_{2}$ sensing coils, the solution of the lift-off variation can be derived by solving the integration equation (15). However, due to the calculation burden caused by the integral from (16) to (19), the processing time is considerable and cannot be applied for the real-time measurement. Besides, the exponential term is illposed to $l_{0}$, which could influence the convergence and accuracy.

\section{Simplified material-independent algorithm}

In Fig. 3, to simplify the integrand and work out the integral, it has been found $\mathrm{M}_{2}$ in $\mathrm{F}_{2}$ and $\mathrm{Y}_{2}$ can be well estimated as a sinusoidal function $\mathrm{e}^{-\alpha(4 \mathrm{~h}} \quad \sin ^{2}\left(\alpha \pi / 2 \alpha_{0}\right) . \alpha_{0}$ controls the peak of the sinusoidal function, which is related to the parameter of the sensor (including the radius, coil height $h$, and separation distance $\mathrm{g}$, according to equation 5)

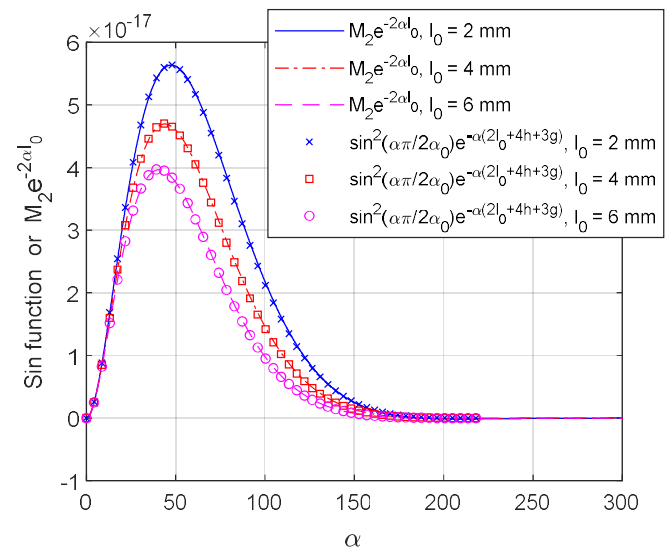

Fig. 3 Estimation of $\mathrm{M}_{2}$ with a sinusoidal function

Since $M_{1}$ cannot be perfectly fitted by the sinusoidal function, only the inductance from the transmitter-reference sensing winding $-\mathrm{L}_{2}$ is considered for the simplified algorithm of lift-off reconstruction.

From the approximation of $\mathrm{M}_{2}$ using the sinusoidal function, the inductance change from the transmitter-reference sensing winding $-\mathrm{L}_{2}$ becomes,

$$
\begin{aligned}
& L_{2}(f)=K G \int_{0}^{2 \alpha_{0}} e^{-\alpha\left(2 l_{0}+4 h+3 g\right)} \sin ^{2}\left(\frac{\alpha \pi}{2 \alpha_{0}}\right)\left(\sqrt{\frac{\mu_{1}}{\pi \sigma \mu_{0} f}} \alpha-1\right) d \alpha \\
& =K\left(\sqrt{\frac{\mu_{1}}{\pi \sigma \mu_{0} f}} Y_{2}-F_{2}\right)
\end{aligned}
$$

In (20), $\mathrm{G}$ is the normalization term between $\mathrm{M}_{2}$ and the sinusoidal function.

$$
G=\frac{P_{t} P_{r}}{\alpha_{0}^{6}} e^{\alpha h}\left(e^{-\alpha h}-1\right)^{2}
$$

Thus, the simplified version of (17) and (19) for (20) becomes,

$$
\begin{aligned}
& F_{2}=G \int_{0}^{2 \alpha_{0}} e^{-\alpha\left(2 l_{0}+4 h+3 g\right)} \sin ^{2}\left(\frac{\alpha \pi}{2 \alpha_{0}}\right) d \alpha \\
& Y_{2}=G \int_{0}^{2 \alpha_{0}} \alpha e^{-\alpha\left(2 l_{0}+2 h+g\right)} \sin ^{2}\left(\frac{\alpha \pi}{2 \alpha_{0}}\right) d \alpha
\end{aligned}
$$

To eliminate the material-dependent term $\sqrt{\frac{\mu_{1}}{\pi \sigma \mu_{0} f}} Y_{2}$, the dual working frequencies $\mathrm{f}_{1}$ and $\mathrm{f}_{2}$ are considered.

$$
\sqrt{\mathrm{f}_{2}} \mathrm{~L}_{2}\left(\mathrm{f}_{2}\right)-\sqrt{\mathrm{f}_{1}} \mathrm{~L}_{2}\left(\mathrm{f}_{1}\right)=\mathrm{K}\left(\sqrt{\mathrm{f}_{1}}-\sqrt{\mathrm{f}_{2}}\right) \mathrm{F}_{2}
$$

In (22) and (23), assign,

$$
\mathrm{X}_{2}=\alpha_{0}\left(2 \mathrm{l}_{0}+4 \mathrm{~h}+3 \mathrm{~g}\right)
$$

Then, after the integration, $\mathrm{F}_{2}$ becomes,

$$
F_{2}=\frac{\pi^{2} \alpha_{0} G\left(1-e^{-2 X_{2}}\right)}{2 X_{2}\left(X_{2}^{2}+\pi^{2}\right)}
$$

Since $X_{2} \gg 1$, the exponential term $e^{-2 X_{2}} \ll 1$. Thus, $F_{2}$ becomes,

$$
F_{2}=\frac{\pi^{2} \alpha_{0} G}{2 X_{2}\left(X_{2}^{2}+\pi^{2}\right)}
$$

Substitute (27) into (24), a simplified equation (without integration) for $\mathrm{X}_{2}$ can be derived.

$$
\frac{\pi^{2} \alpha_{0} G}{2 X_{2}\left(X_{2}^{2}+\pi^{2}\right)}=\frac{\sqrt{f_{2}} L_{2}\left(f_{2}\right)-\sqrt{f_{1}} L_{2}\left(f_{1}\right)}{K\left(\sqrt{f_{1}}-\sqrt{f_{2}}\right)}
$$

Assume the solution of $\mathrm{X}_{2}$ in the simplified equation (28) is $\mathrm{X}_{\mathrm{s}}$, then the lift-off distance can be derived according to equation (2).

$$
\mathrm{l}_{0}=\frac{\mathrm{X}_{\mathrm{s}}}{2 \alpha_{0}}-\frac{4 \mathrm{~h}+3 \mathrm{~g}}{2}
$$

D. Lift-off insensitive inductance feature-measurement of relative magnetic permeability

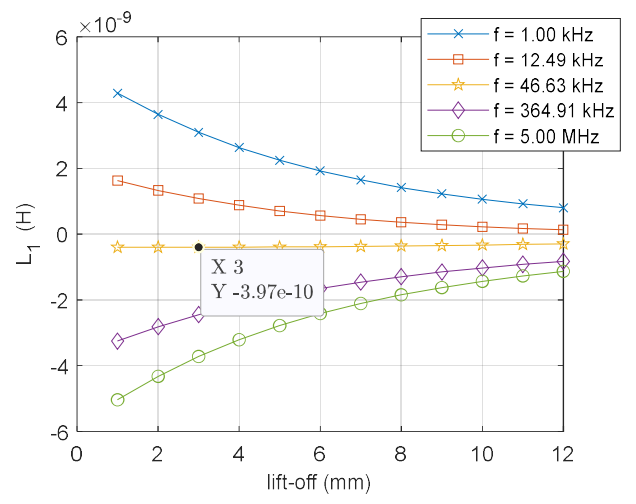

(a) 


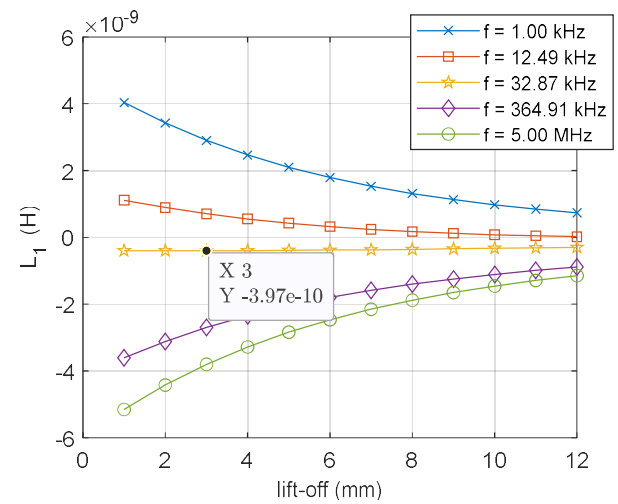

(b)

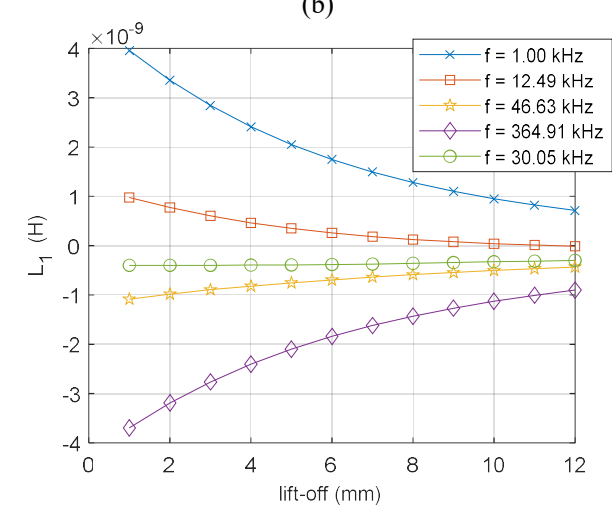

(c)

Fig. 4 Analytical inductance change from the transmitter-receiver sensing pair versus lift-off distance under different working frequencies a) DP $600 \mathrm{~b}$ ) DP 800 c) Duplex stainless steel (in Table 2)

Since the transmitter-receiver sensing pair is closer and sensitive to the test material (compared to transmitterreference), its inductance value is used for the measurement of the magnetic permeability of the magnetic slab. As can be observed in Fig. 4, the inductance change $L_{1}$ is found insensitive to the lift-off variation under a certain working frequency. Besides, the corresponding inductance is independent of different materials. For the sensor listed in Table 1 , the corresponding inductance, termed as the lift-off insensitive inductance, is $-3.97 \times 10^{-10} \mathrm{H}$. Therefore, the magnetic permeability can be measured by combining the derived lift-off distance from the transmitter-reference coil via (29) with the corresponded frequency at the lift-off insensitive inductance. The relative magnetic permeability can be restored from iterative loops [24].

$$
\mu_{1}=\Delta \mu_{1}+\mu_{\mathrm{r}}
$$

In (30), $\mu_{r}$ is the reference relative magnetic permeability. The change of the relative permeability in each iterative loop is,

$$
\Delta \mu_{1}=\mathrm{J}^{-1}\left(\mathrm{~L}_{0}-\mathrm{L}_{1}\left(\mu_{\mathrm{r}}, \mathrm{f}_{\mathrm{L}_{0}}\right)\right)
$$

$L_{1}$ is the analytical inductance with the input of $\mu_{\mathrm{r}}$ and the corresponded frequency $\mathrm{f}_{\mathrm{L}_{0}}$ at $\mathrm{L}_{0}$. That is, the input frequency of $\mathrm{L}_{1}$ can be referred from the frequency (according to the swept-frequency inductance spectrum) closest to the lift-off insensitive inductance $\mathrm{L}_{0}$. J is the inductance sensitivity around $\mu_{\mathrm{r}}$.

$$
J=\frac{L_{1}\left(\mu_{r}, f_{L_{0}}\right)-L_{1}\left(\mu_{r}-\lambda \mu_{r}, f_{L_{0}}\right)}{\lambda \mu_{r}}
$$

$\lambda$ in (32) is a residual value, which is defined as 0.01 for the restoration loop.

\section{EXPERIMENT}

The predictions algorithms in (29) and (30) have been tested with measurements on three different materials of magnetic steels. The eddy current sensor is designed as three co-axial coil windings (structure shown in Fig. 1). The transmitter is enwound between the receiver (bottom) coil and reference (top) coil with an identical separation gap g. As listed in Table 1, the radius of the receiver and the reference coil is designed larger than that of the transmitter coil to fully receive the reflected magnetic flux from the test piece. The measured inductance from the transmitter-reference sensing pair is used for the inversion of the lift-off distance via the simplified materialindependent algorithm in (28) and (29). Besides, the corresponding frequency of the measured inductance (from transmitter-receiver sensing pair) closest to the benchmark (liftoff insensitive inductance) is used for the inversion of the magnetic permeability of the samples.

TABLE I

PARAMETERS OF THE EDDY CURRENT SENSOR

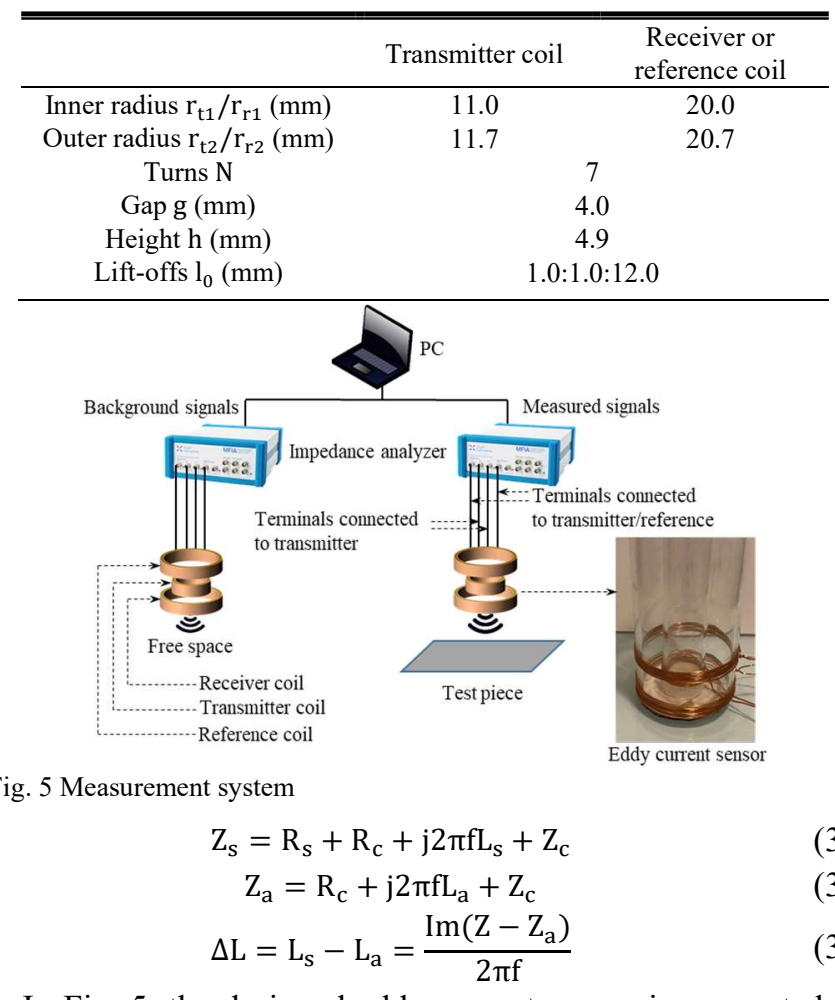

In Fig. 5, the designed eddy current sensor is connected to the impedance analyser. The measured inductance data is exported to the PC via the USB interface cable. In equations from (33) to (35), $Z_{s}$ and $Z_{a}$ denote the measured impedance with and without (in the free space) the sample, respectively; $L_{s}$ and $\mathrm{L}_{\mathrm{a}}$ are the experimental inductance with and without (in the free space) the sample, respectively; $R_{s}$ and $R_{c}$ are the mutual resistance (real part of the impedance) caused by the sample and coils, respectively; By using the inductance change equation in (35) (which is corresponding to the analytical equations in 1 and 2 ), the ambient noise signals (including the mutual resistance $R_{s}$ and $R_{c}$, or potentially high-frequency parasitic impedance of the coils $Z_{c}$ [35]) are excluded from the experimental inductance change. Besides, the working frequency of the 
impedance analyser is from $1 \mathrm{kHz}$ to $5 \mathrm{MHz}$, which is much lower than the resonance frequency. Consequently, the proximity effect (with parasitic capacitance) barely exists during the measurement. Frequencies lower than $1 \mathrm{kHz}$ will result in a relatively poor Signal-to-Noise Ratio (SNR).

As listed in Table 2, the magnetic steels are (ferrite-austenite) alloys with different ferrite fractions. Since the thickness of the steel slab is much larger than the skin depth, the samples can be treated as the half-space (and the skin effect exists) under the whole frequency range. Therefore, the phase term $\Phi$ in (8) can be approximated by (10), which is independent of the sample thickness.

TABLE II

PARAMETERS OF MAGNETIC STEELS

\begin{tabular}{cccc}
\hline \hline & DP 600 & DP 800 & Duplex stainless steel \\
\hline Thickness (mm) c & 4.0 & 4.3 & 6.45 \\
Relative permeability $\mu_{1}$ & 222 & 144 & 45 \\
$\begin{array}{c}\text { Electrical conductivity } \sigma \\
(\mathrm{MS} / \mathrm{m})\end{array}$ & 4.13 & 3.80 & 1.30 \\
\hline
\end{tabular}

\section{RESULT AND DISCUSSION}

\section{A. Swept-frequency inductance}

The swept-frequency inductance change (due to the test steel) from both the transmitter-receiver and transmitterreference sensing pairs are shown in Fig. 6 a) and b). As the frequency increases, the inductance curve begins with a positive value then gradually decreases to a negative one. Besides, the inductance curve of one sample with different lift-off distances will converge at one point, where the measured inductance is shown less affected by the lift-off distance. As the frequency further increases, the inductance curve of one sample with different lift-off variations will gradually diverge. However, the inductance curve for one lift-off distance but different samples will gradually converge, where the inductance is shown sensitive to the lift-off distance but less sensitive to the test sample due to the restrained eddy current under the skin effect. Thus, the lift-off distance of the sensor is inversed from the inductance under high working frequencies.

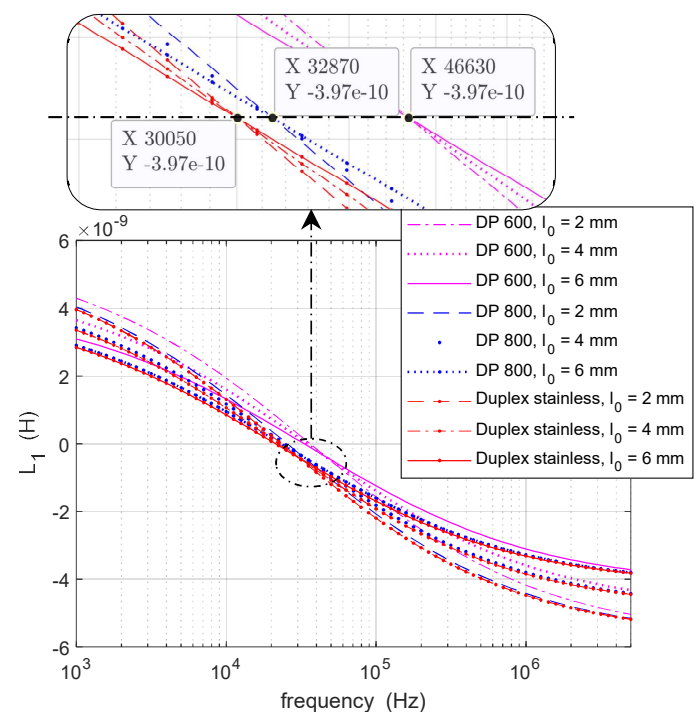

(a)

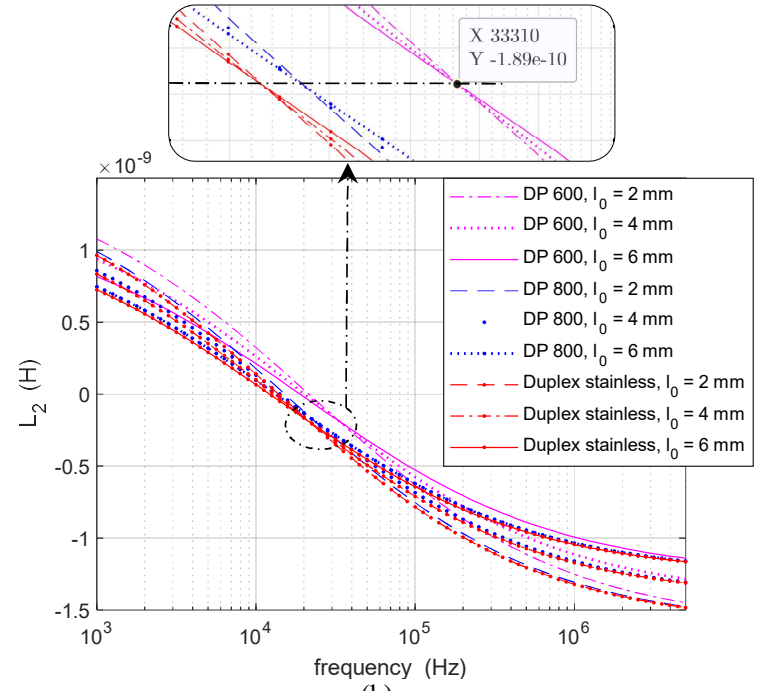

(b)

Fig. 6 Swept-frequency inductance for the sensor above the magnetic slab with lift-off distance of 2, 4, and $6 \mathrm{~mm}$ a) transmitter-receiver sensing pair b) transmitter-reference sensing pair

\section{B. Inversion of lift-off distance using simplified material- independent algorithm}

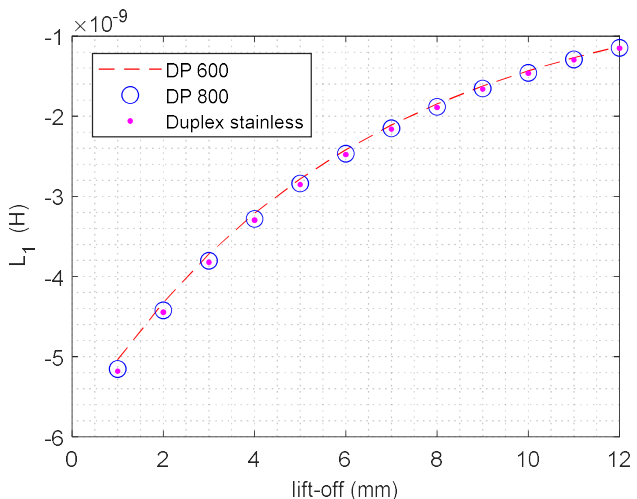

(a)

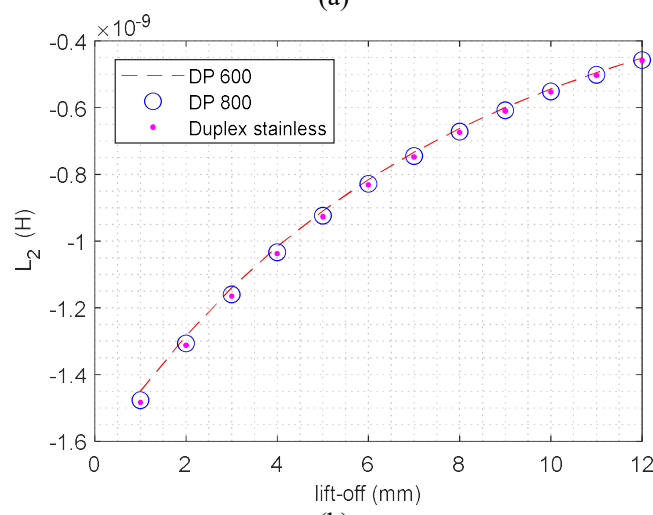

(b)

Fig. 7 Inductance for the sensor above the magnetic slab with different lift-off distances under the working frequency of $5 \mathrm{MHz}$ a) transmitter-receiver sensing pair b) transmitter-reference sensing pair

Fig. 7 shows the inductance change from both the transmitter-receiver and transmitter-reference sensing pairs at different lift-off distances under the working frequency of 5 MHz. It can be observed that, due to the significantly restrained eddy current under the skin effect, the inductance of different lift-off distances is less influenced by the test steel, particularly when the lift-off distance reaches $12 \mathrm{~mm}$. As shown in Fig. 2, 
under the high working frequencies, the material-dependent phase term $\Phi$ approaches -1. Thus, with the increased working frequency, the test steel gradually becomes a pure inductive material. Besides, the inductance is less affected by the parameters of the material, as can be referred to equation (12).

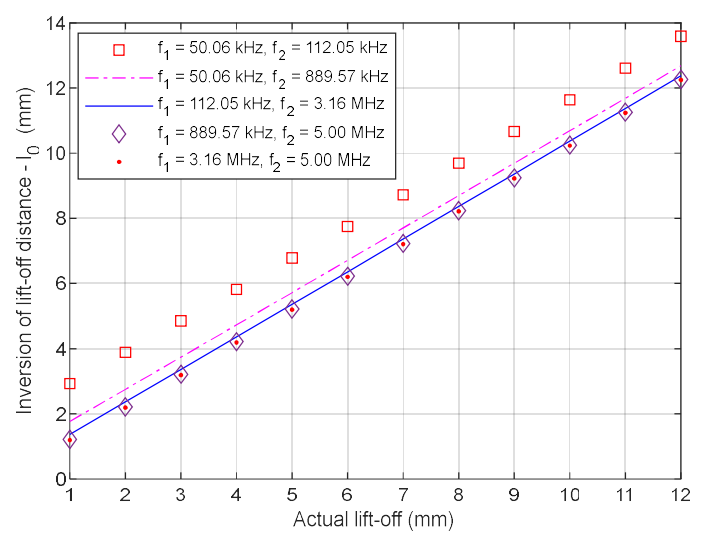

(a)

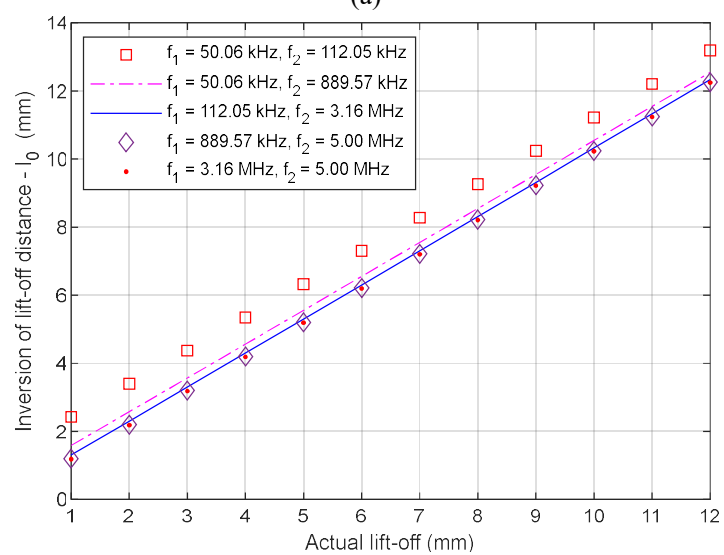

(b)

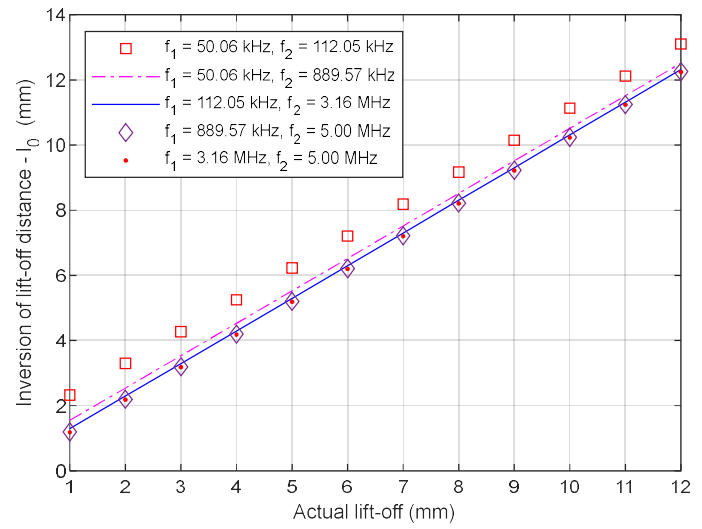

(c)

Fig. 8 Inversion of the lift-off distance from the inductance of different dualfrequency combinations when the test piece is a) DP 600 b) DP 600 c) Duplex stainless steel

As only the coil-dependent magnitude term $\mathrm{M}_{2}$ for transmitter-reference sensing pair can be well fitted by the sinusoidal function, the lift-off distance is inversed from $\mathrm{L}_{2}$ using the simplified algorithm in (28) and (29). As shown in Fig. 8, with the inductance change of dual working frequencies, the inversed lift-off distance is shown less affected by different samples, particularly for the dual-frequency combinations of
889.57 kHz - 5.00 MHz and 3.16 MHz - 5.00 MHz (can be referred to Fig. 9 - the error of the lift-off inversion).

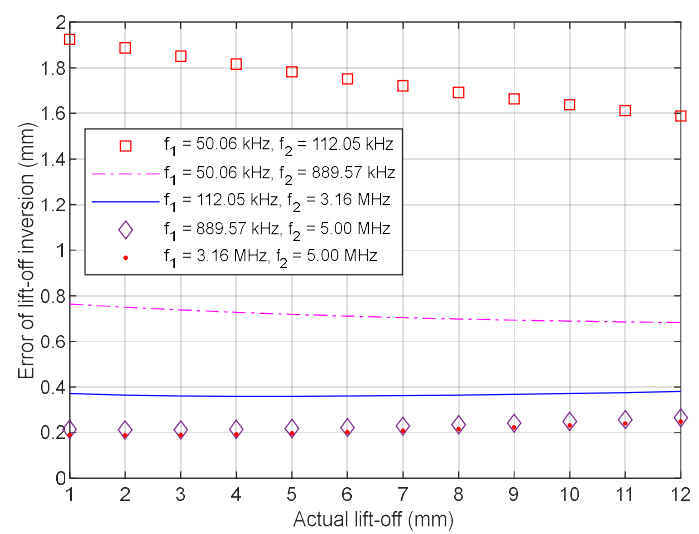

(a)

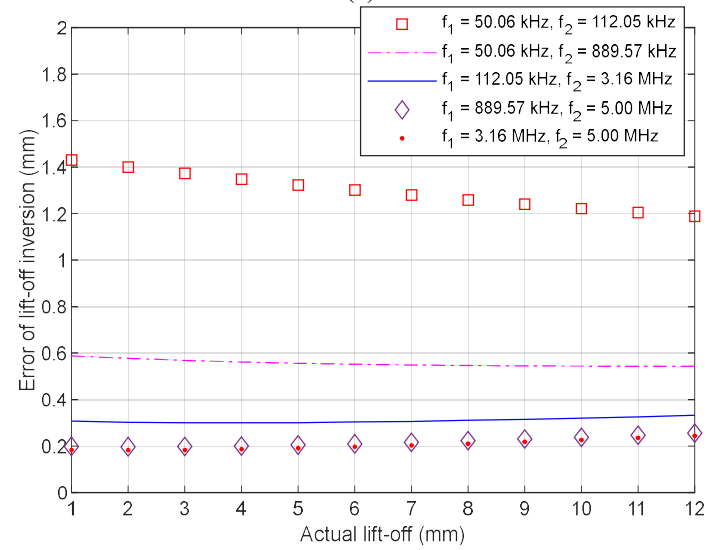

(b)

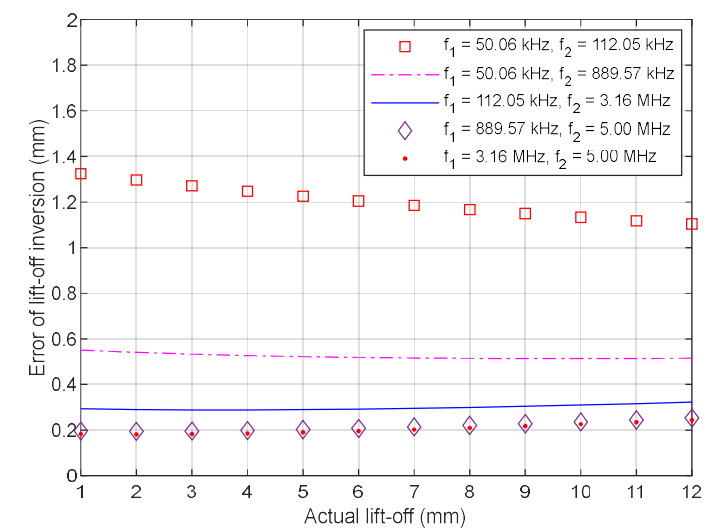

(c)

Fig. 9 Error of the inversion for the lift-off distance from the inductance of different dual-frequency combinations when the test piece is a) DP 600 b) DP 600 c) Duplex stainless steel

In Fig. 9, the lift-off inversion is shown to be more affected by different samples under low dual-frequency combinations, especially for the dual-frequency combination of $50.06 \mathrm{kHz}-$ $112.05 \mathrm{kHz}$, where the phase term $\Phi$ in (12) (Fig. 2) is more influenced by the parameter $\mu_{1}$ and $\sigma$. Considering the accuracy, the inversed lift-off under the dual-frequency combinations of $3.16 \mathrm{MHz}-5.00 \mathrm{MHz}$ is selected for the further inversion of the magnetic permeability (with a maximum error of $0.24 \mathrm{~mm}$ ). 
C. Inversion of magnetic permeability based on the feature lift-off insensitive inductance

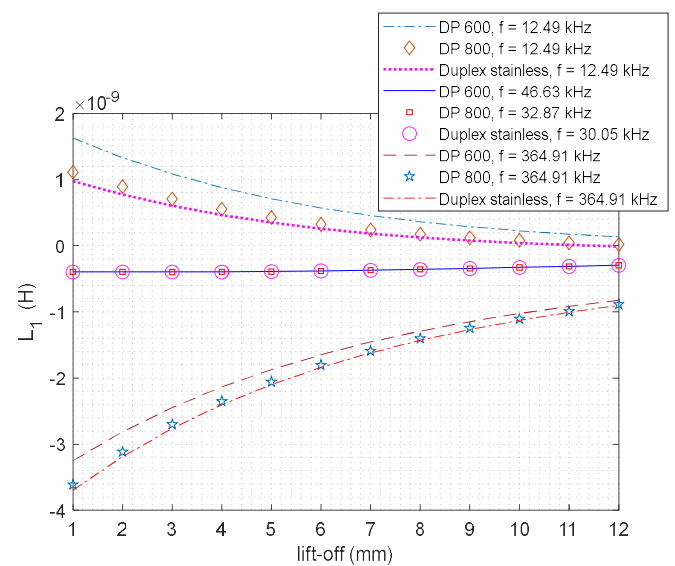

Fig. 10 Inductance for the transmitter-receiver sensing pair above the magnetic slab versus lift-off distance under different working frequencies

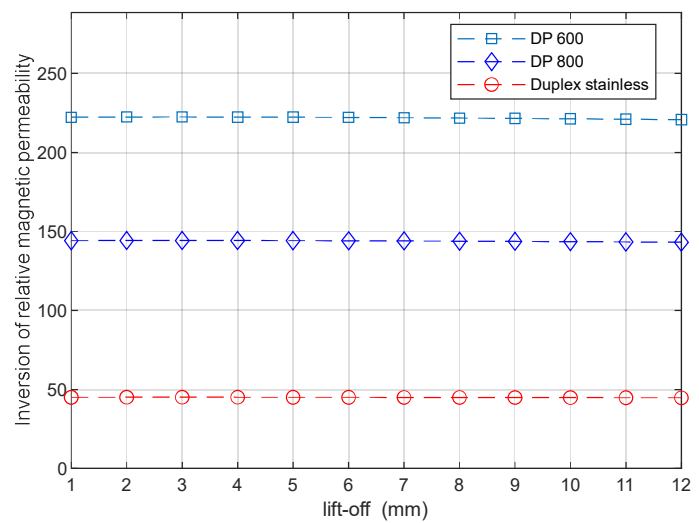

(a)

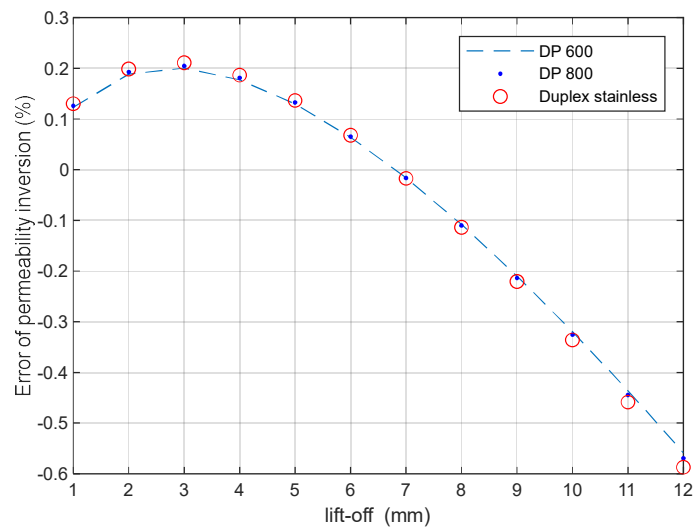

(b)

Fig. 11 a) Inversion of the relative magnetic permeability versus lift-off distance under the corresponded frequency of the lift-off insensitive inductance b) error of the inversion

As the transmitter-receiver sensing pair is closer and more sensitive to the test steel, the inductance $L_{1}$ is used for the inversion of the relative magnetic permeability. Fig. 10 depicts $\mathrm{L}_{1}$ versus lift-off distances under different working frequencies. It can be observed that for one sample, the inductance change is shown to be less affected by the lift-off distance, termed as the lift-off insensitive inductance. Moreover, inductance curves of different samples share the same lift-off insensitive inductance $\mathrm{L}_{0}$ (around $-3.97 \times 10^{-10} \mathrm{H}$ ). From the swept- frequency inductance in Fig. 6 a), the corresponding frequency of the inductance closest to the lift-off insensitive inductance $\mathrm{L}_{0}$ are $46.63,32.87$, and $27.78 \mathrm{kHz}$ for DP 600, DP 800, and Duplex stainless steel.

Parameters including the inversed lift-off distance, lift-off insensitive inductance $\mathrm{L}_{0}$ and its corresponded frequency are used for the inversion of the relative magnetic permeability of the steel using iterative equations from (30) to (32). In Fig. 11, with the inversed lift-off distance (material-independent), liftoff insensitive inductance benchmark $\left(\mathrm{L}_{0}=-3.972 \times 10^{-1} \mathrm{H}-\right.$ data tips in in Fig. 10), and corresponded frequencies $\mathrm{f}_{\mathrm{L}_{0}}$ (46.63, $32.87,27,78 \mathrm{kHz}$ for DP 600 , DP 800 , and Duplex stainless steel - legend of Fig. 10 or data tips in Fig. 6), the relative magnetic permeability can be accurately restored with a maximum error of $0.6 \%$ at the lift-off distance of $12 \mathrm{~mm}$. Besides, the error is nearly independent of different ferriteaustinite steels.

\section{CONCLUSION}

In this paper, two algorithms have been proposed for the inversion of both the lift-off distance and magnetic permeability of the steel. For the inversion of the lift-off distance, a simplified algorithm (without redundant integration) has been explored, which therefore can be used for the online measurement. With the inductance of transmitter-reference sensing pair, the inversed lift-off distance is shown to be material-independent. From the experiments on three different magnetic alloys, the lift-off distance is verified can accurately restore the lift-off distance, with a maximum error of $0.24 \mathrm{~mm}$ at $12 \mathrm{~mm}$. Moreover, the restored lift-off distance is implemented for the inversion of magnetic permeability using iterative algorithms. Based on the lift-off insensitive inductance feature (which is independent of the test sample and less affected by the lift-off) of the transmitter-receiver sensing pair (closer and sensitive to the test piece) and its corresponded frequency (sensitive to sample and insensitive to lift-off distance), the error of the restored permeability can be controlled within $0.6 \%$ for the lift-off distance up to $12 \mathrm{~mm}$.

\section{ACKNOWLEDGEMENT}

This work was supported by [UK Engineering and Physical Sciences Research Council (EPSRC)] [grant number: EP/P027237/1] [title: Real-time In-line Microstructural Engineering (RIME)].

Author Contributions: (Methodology, manuscript drafting) M. Lu and X. Meng; (conceptualization, manuscript revision) M. Lu; (experiment data curation, manuscript review) M. Lu, R. Huang, L. Chen; (Supervision) W. Yin, A. Peyton, M. Lu. All authors have read and agreed to the published version of the manuscript.

\section{REFERENCES}

[1]. G. Yang, G. Dib, L. Udpa, A. Tamburrino and S. S. Udpa, "Rotating Field EC-GMR Sensor for Crack Detection at Fastener Site in Layered Structures," IEEE Sensors Journal, vol. 15, no. 1, pp. 463-470, Jan. 2015.

[2]. X. Chen and Y. Lei, "Electrical conductivity measurement of ferromagnetic metallic materials using pulsed eddy current method," NDT \& E International, vol. 75, pp. 33-38, 2015.

[3]. D. Vasic, V. Bilas and D. Ambrus, "Pulsed eddy-current nondestructive testing of ferromagnetic tubes," IEEE Transactions on Instrumentation and Measurement, vol. 53, no. 4, pp. 1289-1294, Aug. 2004. 
[4]. S. J. Dickinson, R. Binns, W. Yin, C. Davis and A. J. Peyton, "The development of a multifrequency electromagnetic instrument for monitoring the phase transformation of hot strip steel," IEEE Transactions on Instrumentation and Measurement, vol. 56, no. 3, pp. 879-886, 2007.

[5]. A. V. Egorov, V. V. Polyakov, D. S. Salita, E. A. Kolubaev, S. G. Psakhie, A. G. Chernyavskii and I. V. Vorobei, "Inspection of aluminum alloys by a multi-frequency eddy current method," Defence Technology, vol. 11, no. 2, pp. 99-103, 2015.

[6]. T. Theodoulidis and E. E. Kriezis, "Eddy current canonical problems (with applications to nondestructive evaluation)", Tech Science Press, USA, no. 0-9717880-1-4, 2006.

[7]. Y. He, G. Tian, H. Zhang, M. Alamin, A. Simm and P. Jackson, "Steel Corrosion Characterization Using Pulsed Eddy Current Systems," IEEE Sensors Journal, vol. 12, no. 6, pp. 2113-2120, June 2012.

[8]. M. Lu, et al., "Acceleration of frequency sweeping in eddy-current computation," IEEE Transactions on Magnetics, vol. 53, pp. 1-8, 2017.

[9]. Y. Shin, D. Choi, Y. Kim and S. Lee, "Signal characteristics of differential-pulsed eddy current sensors in the evaluation of plate thickness," NDT \& E International, vol. 42, no. 3, pp. 215-221, 2009.

[10]. C. C. Tai, J. H. Rose and J. C. Moulder, "Thickness and conductivity of metallic layers from pulsed eddy-current measurements," Review of Scientific Instruments, vol. 67, no. 11, Aug. 1996.

[11]. J. R. Bowler, S. J. Norton and D. J. Harrison, "Eddy-current interaction with an ideal crack. II. The inverse problem," Journal of applied physics, VOL. 75, no. 12, PP. 8138-8144, 1994.

[12]. J. Kral, R. Smid, H. M. G. Ramos and A. L. Ribeiro, "The lift-off effect in eddy currents on thickness modeling and measurement," IEEE Transactions on Instrumentation and Measurement, vol. 62, no. 7, pp. 2043-2049, 2013.

[13]. R. K. Amineh, et al, "Removal of probe lift-off effects on crack detection and sizing in metals by the AC field measurement technique," IEEE Trans. Magnetics, vol. 44, no. 8, pp. 2066-2073, 2008.

[14]. G. Tytko and L. Dziczkowski, "E-Cored Coil With a Circular Air Gap Inside the Core Column Used in Eddy Current Testing," IEEE Transactions on Magnetics, vol. 51, no. 9, pp. 1-4, Sept. 2015.

[15]. A. L. Ribeiro, H. G. Ramos, J. C. Arez, "Liftoff insensitive thickness measurement of aluminum plates using harmonic eddy current excitation and a GMR sensor," Measurement, vol. 45, no. 9, pp. 2246-2253, 2012.

[16]. S. Giguere, B. A. Lepine, and J. M. S. Dubois. "Pulsed eddy current technology: Characterizing material loss with gap and lift-off variations," Journal of Research in Nondestructive Evaluation, vol. 13, no. 3, pp. 119129,2001

[17]. G. Y. Tian and A. Sophian, "Reduction of lift-off effects for pulsed eddy current NDT," NDT \& E International, vol. 38, no. 4, pp. 319-324, 2005.

[18]. B. A. Abu-Nabah, "Reduction of lift-off effect in high-frequency apparent eddy current conductivity spectroscopy," Measurement Science and Technology, vol. 28, no.5, pp. 055107, 2017

[19]. W. Yin, et al., "Analysis of the liftoff effect of phase spectra for eddy current sensors," IEEE Transactions on Instrumentation and Measurement, vol. 56, no. 6, pp. 2775-2781, 2007.

[20]. W. Yin, X. J. Hao, A. Peyton, et al., "Measurement of permeability and ferrite/austenite phase fraction using a multi-frequency electromagnetic sensor," NDT \& E International, vol. 42, no. 1, pp. 6-68, 2009

[21]. E. Pinotti and E. Puppin, "Simple Lock-In Technique for Thickness Measurement of Metallic Plates," IEEE Transactions on Instrumentation and Measurement, vol. 63, no. 2, pp. 479-484, Feb. 2014.

[22]. M. Lu, R. Huang, W. Yin, Q. Zhao and A. Peyton, "Measurement of permeability for ferrous metallic plates using a novel lift-off compensation technique on phase signature," IEEE Sensors Journal, vol. 19, no. 17, pp. 7440-7446, 1 Sept.1, 2019.

[23]. M. Lu, H. Xu, W. Zhu, L. Yin, et al., "Conductivity Lift-off Invariance and measurement of permeability for ferrite metallic plates," NDT \& E International, vol. 95, pp. 36-44, Apr. 2018

[24]. M. Lu, et al., "Determination of the magnetic permeability, electrical conductivity, and thickness of ferrite metallic plates using a multifrequency electromagnetic sensing system," IEEE Transactions on Industrial Informatics, vol. 15, pp. 4111-4119, 2019.
[25]. J. R. S. Avila, M. Lu, et al., "Accurate measurements of plate thickness with variable lift-off using a combined inductive and capacitive sensor," NDT \& E International, vol. 110, pp. 102202, 2020

[26]. M. Lu, L. Yin, A. J. Peyton and W. Yin, "A novel compensation algorithm for thickness measurement immune to lift-off variations using eddy current method," IEEE Transactions on Instrumentation and Measurement, vol. 65, no. 12, pp. 2773-2779, Dec. 2016.

[27]. M. Lu, X. Meng, W. Yin, Z. Qu, F. Wu, J. Tang, et al., "Thickness measurement of non-magnetic steel plates using a novel planar triple-coil sensor," NDT \& E International, vol. 107, 2019.

[28]. R. Huang, M. Lu, A. Peyton and W. Yin, "Thickness measurement of metallic plates with finite planar dimension using eddy current method," IEEE Transactions on Instrumentation and Measurement, vol. 69, no. 10, pp. 8424-8431, 2020.

[29]. M. Lu, et al., "Measurement of ferromagnetic slabs permeability based on a novel planar triple-coil sensor," IEEE Sensors Journal, vol. 20, no. 6, pp. 2904-2910, 2020.

[30]. M. Lu, W. Zhu, L. Yin, A. J. Peyton, W. Yin and Z. Qu, "Reducing the lift-off effect on permeability measurement for magnetic plates from multifrequency induction data," IEEE Transactions on Instrumentation and Measurement, vol. 67, no. 1, pp. 167-174, Jan. 2018.

[31]. C. V. Dodd and W. E. Deeds, "Analytical solutions to eddy-current probe-coil problems," Journal of applied physics, vol. 39, no. 6, pp. 2829-2838, 1968.

[32]. M. S. Luloff, J. Morelli and T. W. Krause, "Examination of Dodd and Deeds solutions for a transmit-receive eddy current probe above a layered planar structure," AIP Conference Proceedings. AIP Publishing LLC, vol. 1806, no. 1, 2017.

[33]. M. Fan, B. Cao, P. Yang, et al., "Elimination of liftoff effect using a model-based method for eddy current characterization of a plate," NDT \& E International, vol. 74, pp. 66-71, 2015.

[34]. J. Tkocz et al., "High power phased EMAT arrays for nondestructive testing of as-cast steel," NDT \& E International, vol. 102, pp. 47-55, 2019.

[35]. M. Lu, et al., "Thickness measurement of metallic film based on a highfrequency feature of triple-coil electromagnetic eddy current sensor," IEEE Transactions on Instrumentation and Measurement, early access, 2020. Doi: 10.1109/TIM.2020.3027929.

[36]. M. Lu, et al., "Measuring lift-off distance and electromagnetic property of metal using dual-frequency linearity feature," IEEE Transactions on Instrumentation and Measurement, early access, 2020. Doi: 10.1109/TIM.2020.3029348.

[37]. M. Lu, et al., "Lift-off tolerant pancake eddy-current sensor for the thickness and spacing measurement of nonmagnetic plates," IEEE Transactions on Instrumentation and Measurement, early access, 2020. DOI: 10.1109/TIM.2020.3033377

[38]. W. Yin, J. Tang, M. Lu, et al., "An equivalent-effect phenomenon in eddy current non-destructive testing of thin structures," IEEE Access, vol. 7, pp. 70296-70307, 2019.

[39]. R. Huang et al., "Measuring Co-Axial Hole Size of Finite-Size Metallic Disk Based on a Dual-Constraint Integration Feature Using MultiFrequency Eddy Current Testing," IEEE Transactions on Instrumentation and Measurement, early access, 2020. DOI: 10.1109/TIM.2020.3026762

[40]. R. Huang et al., "Measurement of the radius of metallic plates based on a novel finite region eigenfunction expansion (FREE) method," IEEE Sensors Journal, early access, 2020. DOI: 10.1109/JSEN.2020.3009443

[41]. J. Tang et al., "A Novel Efficient FEM Thin Shell Model for BioImpedance Analysis," Biosensors, vol. 10, no. 6, pp. 69, 2020.

[42]. L. Chen, et al., "Textile Based Capacitive Sensor for Physical Rehabilitation via Surface Topological Modification," ACS Nano, vol. 14, no. 7, pp. 8191-8201, 2020. DOI: 10.1021/acsnano.0c01643

[43]. Z. Jin, et al., "Methods of Controlling Lift-off in Conductivity Invariance Phenomenon for Eddy Current Testing," IEEE ACCESS, vol. 8, pp. 2169-3536, 2020. DOI: 10.1109/ACCESS.2020.3007216.

[44]. J. Tang, et al., "Effect of frozen-thaw injury on cell membrane and bioimpedance," In 2020 IEEE International Instrumentation and Measurement Technology Conference (I2MTC), pp. 1-6. IEEE, 2020. 
[45]. J. Tang, et al., "Bio-impedance spectroscopy for frozen-thaw of biosamples: Non-contact inductive measurement and finite element (FE) based cell modelling," Journal of Food Engineering, vol. 272, pp. 109784, 2020.

[46]. H. Xu et al., "Imaging a weld cross-section using a novel frequency feature in multi-frequency eddy current testing," Insight-NonDestructive Testing and Condition Monitoring, vol. 61, no. 12, pp. 738 743, 2019.

[47]. Y. Xie et al., "Novel Wearable Sensors for Biomechanical Movement Monitoring Based on Electromagnetic Sensing Techniques," IEEE Sensors Journal, vol. 20, no. 2, 2020. DOI: 10.1109/JSEN.2019.2943487

[48]. H. Xu et al., "Permeability invariance phenomenon and measurement of electrical conductivity for ferrite metallic plates," Insight-NonDestructive Testing and Condition Monitoring, vol. 61, no. 8, pp. 472 479, 2019.

[49]. M. Lu et al., "A model for the triboelectric nanogenerator with inductive load and its energy boost potential," Nano Energy, vol. 63, pp. 103883, 2019.

[50]. W. Yin et al., "An equivalent-effect phenomenon in eddy current nondestructive testing of thin structures," IEEE ACCESS, vol. 7, pp. 70296 $-70307,2019$.

[51]. M. Lu et al., "Forward solver for deep earth exploration and induction logging using custom built Edge - Element FEM technique," Acta Geologica Sinica, vol. 93, pp. 302-304, 2019.

[52]. L. Chen et al., "Whole System Design of Wearable Magnetic Induction Sensor for Physical Rehabilitation," Advanced Intelligent Systems, vol. 1, no. 1, pp. 1900037, 2019.

[53]. Y. X et al., "A self-powered radio frequency (RF) transmission system based on the combination of triboelectric nanogenerator (TENG) and piezoelectric element for disaster rescue/relief," Nano Energy, vol. 54, pp. 331-340, 2018.

[54]. W. Yin et al., "Custom edge-element FEM solver and its application to eddy-current simulation of realistic $2 \mathrm{M}$-element human brain phantom," Bioelectromagnetics, vol. 39, no. 8, pp. 604-616, 2018.

[55]. L. Yin et al., "Detection of corrosion pits based on an analytically optimised eddy current sensor," Insight-Non-Destructive Testing and Condition Monitoring, vol. 60, no. 10, pp. 561-567, 2018.

[56]. W. Yin et al., "Acceleration of eddy current computation for scanning probes," Insight-Non-Destructive Testing and Condition Monitoring, vol. 60 , no. 10, pp. 547-555, 2018.

[57]. W. Zhou et al., "Three-dimensional electromagnetic mixing models for dual-phase steel microstructures," Applied Sciences, vol. 8, no. 4, pp. 547-555, 2018.

[58]. M. Lu, et al., "Determining the magnetic permeability of ferrite steel strip by a custom inversion method," In Proc. 12th ECNDT, pp. 1-8. 2018.

[59]. J. Tang, et al., "Cellular structure analysis based on magnetic induction finite element method simulations and measurements," bioRxiv, pp. 275271, 2018. DOI: $10.1101 / 275271$

[60]. J.R.S. Avila, et al., "A novel dual modality sensor with sensitivities to permittivity, conductivity, and permeability," IEEE Sensors Journal, vol. 18, no. 1, pp. 356-362, 2017.

[61]. T. Yang, et al., "Level measurement for saline with a small surface area using high frequency electromagnetic sensing technique," Measurement, vol. 101, pp. 118-125, 2017.

[62]. M. Lu, et al., "Prediction of the asymptotical magnetic polarization tensors for cylindrical samples using the boundary element method," In 2015 IEEE Sensors Applications Symposium (SAS), pp. 1-4. IEEE, 2015. 